\title{
Many-body diffusion and path integrals for identical particles
}

\author{
L. F. Lemmens \\ Departement Natuurkunde, Universiteit Antwerpen (RUCA), Groenenborgerlaan 171, B-2020 Antwerpen, Belgium \\ F. Brosens and J. T. Devreese* \\ Departement Natuurkunde, Universiteit Antwerpen (UIA), Universiteitsplein 1, B-2610 Antwerpen, Belgium
}

(Received 25 October 1995)

\begin{abstract}
For distinguishable particles it is well known that Brownian motion and a Feynman-Kac functional can be used to calculate the path integral (for imaginary times) for a general class of scalar potentials. In order to treat identical particles, we exploit the fact that this method separates the problem of the potential, dealt with by the Feynman-Kac functional, from the process which gives sample paths of a noninteracting system. For motion in one dimension, we emphasize that the permutation symmetry of the identical particles completely determines the domain of Brownian motion and the appropriate boundary conditions: absorption for fermions, reflection for bosons. Further analysis of the sample paths for motion in three dimensions allows us to decompose these paths into a superposition of one-dimensional sample paths. This reduction expresses the propagator (and consequently the energy and other thermodynamical quantities) in terms of well-behaved one-dimensional fermion and boson diffusion processes and the Feynman-Kac functional.
\end{abstract}

PACS number(s): 05.30.Fk, 03.65.Ca, 02.50.Ga, 02.70.Lq

\section{INTRODUCTION}

In the present paper we introduce a process that combines multidimensional Brownian motion on domains with appropriate boundary conditions to solve the many-body problem of fermions or bosons interacting through a general class of scalar potentials. This process is a superposition of orthogonal and independent fermion and boson diffusion processes combined in a precise and prescribed way. In combination with the Feynman-Kac functional this approach allows us to write the propagator of the many-body Schrödinger equation as an expectation over the functional along the sample paths generated by this process. Our method extends the well known techniques for quantum models with distinguishable particles [1-6] to quantum models with identical particles, in such a way that the so-called "sign problem" [7-10] for fermions is solved, and that for bosons and fermions sample paths over configurations $[11-15,8]$ generated by the permutation symmetry are avoided. This opens the perspective that with the proper algorithms this process would improve the standard approach [16-22] used in path integral Monte Carlo for fermions as well as for bosons.

Fermion diffusion and boson diffusion are (multidimensional) Brownian motions on an $n$-dimensional domain $D_{n}$. If $x_{1}, x_{2}, \ldots, x_{n}$ denote the possible components of the positions of the particles on a line, the domain $D_{n}$ is defined by the condition $x_{1} \geqslant x_{2} \geqslant \cdots \geqslant x_{n}$ [23]. Brownian motion with reflection at the boundary of $D_{n}$ has been studied in the context of traffic flow models $[24,25]$ and will be identified below with the boson diffusion process, because it leads indeed to the propagator of $n$ noninteracting bosons on a line. Brownian motion with absorption at the boundary of $D_{n}$ has been identified by the present authors $[26,27]$ as the process

\footnotetext{
*Also at Universiteit Antwerpen (RUCA), and Technische Universiteit Eindhoven, Eindhoven, The Netherlands
}

which leads to the propagator of $n$ noninteracting fermions on a line. We have argued that a three-dimensional (3D) extension of this process consisting of fermion diffusion in one direction and standard Brownian motion in the two other directions is sufficient to obtain the ground state energy of an interacting 3D fermion system. The present analysis strongly supports our previous arguments. It should be mentioned that our investigation was originated by Korzeniowski et al. [28] and the subsequent discussions on this work [29-31].

Because of the fact that for 1D problems the stochastic approach to the many-body problem for identical particles could be formulated in terms of a fermion or a boson diffusion process, we concentrated our attention on the reduction of the sample paths of a 3D process to three one-dimensional processes. For the multidimensional Brownian motion of distinguishable particles this reduction is trivial. Indeed, on the level of the process each 3D sample path is made up by $1 \mathrm{D}$ Brownian motions of the components of the process vector. For identical particles, such a reduction is not obvious. But by realizing that the knowledge of the propagator of a noninteracting many-body system of identical particles suffices to study how this reduction to independent $1 \mathrm{D}$ processes can be performed, the character of the many-body diffusion could be revealed. The analysis of this reduction is postponed until Sec. IV and constitutes the main result of the present paper. Before studying this reduction, we first summarize in Sec. II the basic underlying concepts, borrowed from the stochastic approach to the quantum theory of distinguishable particles. In Sec. III the fermion and boson diffusion processes are derived and discussed in some detail.

\section{THE FEYNMAN-KAC FUNCTIONAL FOR DISTINGUISHABLE PARTICLES}

In this section we briefly describe how the Feynman-Kac functional and multidimensional Brownian motion can be used to solve the Schrödinger equation or to obtain the par- 
tition function of a many-body problem with distinguishable particles. The basic equations and the mathematical notations are presented.

\section{A. The 3D configuration space}

For a free particle moving in a 3D space it is well known that the Schrödinger equation becomes a diffusion equation if the real time variable is transformed into an imaginary time variable. This means that the motion of the particle can be represented [4] by a process $\{\vec{X}(t) ; t \geqslant 0\}$ which can take the realization $\vec{x}(t)$. The probability density relating the position $\vec{x}(t)$ to a previous position $\vec{x}^{\prime}\left(t_{0}\right)$ is Gaussian and given by

$$
\rho\left(\vec{x}, t ; \vec{x}^{\prime}, t_{0}\right)=\left(\frac{m}{2 \pi \hbar\left(t-t_{0}\right)}\right)^{3 / 2} \exp \left(-\frac{m\left(\vec{x}-\vec{x}^{\prime}\right)^{2}}{2 \hbar\left(t-t_{0}\right)}\right) .
$$

The process $\{\vec{X}(t) ; t \geqslant 0\}$ is related to standard Brownian motion $\{\vec{B}(t) ; t \geqslant 0\}$ in $3 \mathrm{D}$ with infinitesimal variance $\sigma^{2}=\hbar / m$,

$$
d \vec{X}(t)=\sqrt{\frac{\hbar}{m}} d \vec{B}(t)
$$

If the particle moves in a potential $V(\vec{x})$, the solution of the Schrödinger equation can be obtained as an average over a Feynman-Kac functional. This average is the expectation over the process (2.2). For an arbitrary function $f[\vec{X}(t)]$ of the process, the expectation is defined as [32]

$$
E_{\vec{x}}\{f[\vec{X}(s)]\}=\int d \vec{x}^{\prime} \rho\left(\vec{x}, t+s ; \vec{x}^{\prime}, t\right) f\left(\vec{x}^{\prime}\right) .
$$

Thus the expectation is the average of $f[\vec{X}]$ over all paths that end in the position $\vec{x}$ at time $t+s$, starting at all possible positions $\vec{x}^{\prime}$ at time $t$. If the function to be averaged is defined by an integral over time, the process is constructed in such a way that

$$
E_{\vec{x}}\left\{\int_{0}^{t} g[\vec{X}(s)] d s\right\}=\int_{0}^{t} E_{\vec{x}}\{g[\vec{X}(s)]\} d s .
$$

From the properties of the expectation and the process it is easy to show that for a large class of potentials $V(\vec{x})$ the solution of the "Schrödinger equation", (in imaginary time)

$$
\hbar \frac{\partial}{\partial t} \Psi(\vec{x}, t)=\frac{\hbar^{2}}{2 m} \nabla^{2} \Psi(\vec{x}, t)-V(\vec{x}) \Psi(\vec{x}, t)
$$

is given by

$$
\Psi(\vec{x}, t)=E_{\vec{x}}\left\{f[\vec{X}(t)] \exp \left[-\frac{1}{\hbar} \int_{0}^{t} V(\vec{X}(s)) d s\right]\right\},
$$

where $f[\vec{X}(t)]$ ensures the initial condition on $\Psi(\vec{x}, t)$. If one wants to calculate the propagator of $(2.5)$, this initial condition is

$$
\lim _{t \downarrow 0} \Psi(\vec{x}, t)=\delta\left(\vec{x}-\vec{x}^{\prime}\right)
$$

and hence replacing $f[\vec{X}(t)]$ by a $\delta$ function (2.7), one obtains the propagator

$$
K\left(\vec{x}, t \mid \vec{x}^{\prime}\right)=E_{\vec{x}}\left\{f[\vec{X}(t)] \exp \left[-\frac{1}{\hbar} \int_{0}^{t} V(\vec{X}(s)) d s\right]\right\},
$$

which solves the Schrödinger equation (2.5) and satisfies the initial condition (2.7).

The Brownian motion (2.2) has independent increments in the $x, y$, and $z$ direction. This means that at the level of this process there is no difference between the sample paths of one particle moving in three dimensions, and three particles moving each in one dimension. At the level of the potential the difference between three 1D potentials and one 3D potential is obvious. The $3 \mathrm{D}$ character of the potential and hence of the problem can (at least partly) be transferred to the process by introducing a local drift vector $\vec{\mu}[\vec{X}(t)]$ in the process

$$
d \vec{X}(t)=\vec{\mu}[\vec{X}(t)] d t+\sqrt{\frac{\hbar}{m}} d \vec{B}(t)
$$

and the corresponding modification in the Feynman-Kac functional $[33,34]$. But it is clear that changing the process according to (2.9) only relabels the state space.

\section{B. The $3 n$-dimensional configuration space}

The generalization of the preceding stochastic description of the evolution in the imaginary time domain of a quantum system with three degrees of freedom to a system with $3 n$ degrees of freedom is straightforward. A point of the configuration space given by a $3 n$-dimensional vector $\bar{x}$ represents now a state of the process. For convenience in the treatment below, we use the following labeling (for brevity writing down the row vector $\vec{x}^{T}$ ):

$$
x^{-T}=\left(x_{1}, y_{1}, z_{1}, x_{2}, y_{2}, z_{2}, \ldots, x_{n}, y_{n}, z_{n}\right) .
$$

The process $\{\bar{X}(t) ; t \geqslant 0\}$ with realization $\bar{x}(t)$ at time $t$ is a configuration obtained according to a straightforward extension of (2.2):

$$
d \bar{X}(t)=\sqrt{\frac{\hbar}{m}} d \bar{B}(t)
$$

where $\{\bar{B}(t) ; t \geqslant 0\}$ is the $3 n$-dimensional Brownian motion. The transition probability density for an increment $(\bar{x}-\vec{x})$ in a time lapse $s$ for this process is given by

$$
\rho(\bar{x}, t+s ; \vec{x}, t)=\left(\frac{m}{2 \pi \hbar s}\right)^{3 n / 2} \exp \left(-\frac{m[\bar{x}-\vec{x}]^{T}[\bar{x}-\vec{x}]}{2 \hbar s}\right) .
$$

The matrix product in (2.12) can equally well be written as a dot product: 


$$
[\bar{x}-\vec{x}]^{T}[\bar{x}-\vec{x}]=(\bar{x}-\vec{x}) \cdot(\bar{x}-\vec{x})=(\bar{x}-\vec{x})^{2},
$$

but the matrix notation will be more advantageous for our purposes.

For the multidimensional process $\{\bar{X}(t) ; t \geqslant 0\}$ the propagator solution of

$$
\hbar \frac{\partial}{\partial t} \Psi(\bar{x}, t)=\left(\frac{\hbar^{2}}{2 m} \sum_{i=1}^{3 n} \frac{\partial^{2}}{\partial x_{i}^{2}}-V(\bar{x})\right) \Psi(\bar{x}, t)
$$

can again be written as a Feynman-Kac functional:

$$
K(\bar{x}, t \mid \vec{x})=E_{\bar{x}}\left\{f[\bar{X}(t)] \exp \left[-\frac{1}{\hbar} \int_{0}^{t} V(\bar{X}(s)) d s\right]\right\}
$$

if for $f[\bar{X}(t)]$ a $\delta$ function is taken to impose the initial condition $\lim _{t \downarrow 0} K(\bar{x}, t \mid \vec{x})=\delta(\bar{x}-\vec{x})$.

It is clear that a straightforward extension of (2.9) with a drift vector $\bar{\mu}(\bar{X}(t))$ can facilitate the actual calculations, but the formulation of the problem with or without drift is equivalent. Therefore also in the case of $3 n$ degrees of freedom, the process (2.11) that realizes the sample paths for the Feynman-Kac functional does not distinguish between $n$ particles moving in $3 \mathrm{D}$ or $3 n$ particles moving in $1 \mathrm{D}$. This situation will be different in the case of identical particles which will be considered below.

\section{PERMUTATIONS AND PROCESSES}

A striking example of how permutation symmetry can be used to simplify joint probability distributions can be found in order statistics [35]. The basic idea is that for most samples the order in which the values are measured is irrelevant, which means that any permutation of the observed values should have the same probability. This constraint makes the joint probability distribution symmetrically dependent. The dependence can be lifted by reducing the sample space to ordered sample points: thus for an observation $x_{1}, x_{2}, \ldots, x_{n}$ the sample point $x_{(1)}, x_{(2)}, \ldots, x_{(n)}$ is considered where the observed values are ordered in such a way that $x_{(1)} \geqslant x_{(2)} \geqslant \cdots \geqslant x_{(n)}$.

The idea of lifting the dependence implied by the permutation symmetry through a reduction of the domain has been put forward before. The basic ingredient of the Bethe ansatz [37] is precisely the restriction of the positions on a line in such a way that the positions remain ordered. This observation has also been used to solve the nodal plane problem for fermions in one dimension [9]. In particle physics the spatial-temporal distribution of bosons and fermions in beams has been obtained using this reduction scheme applied on point processes [38-41]. For a diffusion process we applied an analogous construction to obtain the propagator of interacting fermions [26]. The process used to realize the sample paths was called the "fermion diffusion process" by the present authors. It is an $n$-dimensional diffusion with the appropriate (absorption) boundary conditions.

We first illustrate the technique for two particles, and summarize the properties of the fermion diffusion process. Subsequently it will be shown how this reduction scheme can also be used to construct the $n$-dimensional boson diffusion process.

\section{A. Two particles on a line}

Let $x_{1}$ and $x_{2}$ be the coordinates of the first and the second particle, respectively. The configuration space is two dimensional: $\left(x_{1}, x_{2}\right) \in R^{2}$. If the particles are identical the configuration $\left(x_{1}, x_{2}\right)$ and the configuration $\left(x_{2}, x_{1}\right)$ should indicate the same state. For fermions with parallel spin, the antisymmetry under the interchange of the two particles is taken into account by the propagator

$$
\begin{aligned}
\left\langle x_{1}, x_{2}\left|e^{-t H / \hbar}\right| x_{1}^{\prime}, x_{2}^{\prime}\right\rangle= & \rho\left(x_{1}, t ; x_{1}^{\prime}, 0\right) \rho\left(x_{2}, t ; x_{2}^{\prime}, 0\right) \\
& -\rho\left(x_{1}, t ; x_{2}^{\prime}, 0\right) \rho\left(x_{1}, t ; x_{2}^{\prime}, 0\right) .
\end{aligned}
$$

We consider this formula for $x_{1} \geqslant x_{2}$ and $x_{1}^{\prime} \geqslant x_{2}^{\prime}$, thus for the position elements of the state space being $\left(x_{1}, x_{2}\right) \in D_{2}$ and $\left(x_{1}^{\prime}, x_{2}^{\prime}\right) \in D_{2}$. The boundary of $D_{2}$ is defined by $x_{1}=x_{2}$ and denoted by $\partial D_{2}$.

It should be noted that the usual factor $1 / 2$ ! in front of the propagator defined over the configuration space does not occur if the motion is restricted to the domain $D_{2}$. Moreover, the propagator on $D_{2}$ has all the properties of a transition probability density of a diffusion process with absorbing boundary conditions: (i) it is positive on $D_{2}$, (ii) it conserves the probability flux if the boundary state is explicitly introduced [42-44], and (iii) it has the semigroup property. These requirements can be checked by direct calculation. The positivity of the propagator can also be understood on the basis of a simple geometrical argument: the distance between $\left(x_{1}, x_{2}\right)$ and $\left(x_{1}^{\prime}, x_{2}^{\prime}\right)$ both in $D_{2}$ is always smaller than the distance between $\left(x_{1}, x_{2}\right)$ and $\left(x_{2}^{\prime}, x_{1}^{\prime}\right)$ except at the boundary $\partial D_{2}$ where both distances are equal. For a Brownian motion this relation between the distances implies that the probability density to go from a point $\left(x_{1}, x_{2}\right)$ in $D_{2}$ to another point $\left(x_{1}^{\prime}, x_{2}^{\prime}\right)$ in $D_{2}$ in a fixed time lapse $t$ is always larger than the probability density to go from the same point $\left(x_{1}, x_{2}\right)$ to the reflected point $\left(x_{2}^{\prime}, x_{1}^{\prime}\right)$ outside $D_{2}$ in the same time lapse.

Having found the diffusion process for two fermions as a two-dimensional diffusion process on $\mathrm{D}_{2}$ with absorption on the boundary - the so-called fermion diffusion process-it is easy to show that similar considerations hold for two bosons, starting from the propagator

$$
\begin{aligned}
\left\langle x_{1}, x_{2}\left|e^{-t H / \hbar}\right| x_{1}^{\prime}, x_{2}^{\prime}\right\rangle= & \rho\left(x_{1}, t ; x_{1}^{\prime}, 0\right) \rho\left(x_{2}, t ; x_{2}^{\prime}, 0\right) \\
& +\rho\left(x_{1}, t ; x_{2}^{\prime}, 0\right) \rho\left(x_{1}, t ; x_{2}^{\prime}, 0\right) .
\end{aligned}
$$

Again the propagator (3.2) is a transition probability density of a two-dimensional diffusion process on $D_{2}$, but now with reflecting boundary conditions on $\partial D_{2}$. Similarly as for fermions, the required conditions for such a transition probability density are easily verified. A nice consequence of the restriction of the state space to the domain $D_{2}$ is that (for both fermions and bosons) the propagator in $D_{2}$ satisfies the initial condition 


$$
\begin{array}{r}
\lim _{t \downarrow 0}\left\langle x_{1}, x_{2}\left|e^{-t H / \hbar}\right| x_{1}^{\prime}, x_{2}^{\prime}\right\rangle=\delta\left(x_{1}-x_{1}^{\prime}\right) \delta\left(x_{2}-x_{2}^{\prime}\right) \\
\text { if }\left\{\begin{array}{c}
\left(x_{1}, x_{2}\right) \in D_{2} \\
\left(x_{1}^{\prime}, x_{2}^{\prime}\right) \in D_{2} .
\end{array}\right.
\end{array}
$$

It is clear that (3.1) and (3.2) can be written, respectively, as a determinant and a permanent

$$
\left\langle x_{1}, x_{2}\left|e^{-t H / \hbar}\right| x_{1}^{\prime}, x_{2}^{\prime}\right\rangle=\left|\begin{array}{ll}
\rho\left(x_{1}, t ; x_{1}^{\prime}, 0\right) & \rho\left(x_{1}, t ; x_{2}^{\prime}, 0\right) \\
\rho\left(x_{1}, t ; x_{2}^{\prime}, 0\right) & \rho\left(x_{2}, t ; x_{2}^{\prime}, 0\right)
\end{array}\right|_{\xi},
$$

where $\xi=+1$ refers to a permanent (bosons) and $\xi=-1$ means a determinant (fermions). This observation allows us to generalize the process for two identical particles to a process for $n$ identical particles moving in one dimension, because the form of the transition probability density will automatically take the boundary conditions into account. The fact that the transition probability density has to be zero at the boundary implies absorption at the boundary for the fermion problem [42-44]. For the boson problem the transition probability density has to be an extremum at the boundary; this implies that its normal derivative at the boundary is zero and as a consequence the boundary condition is a reflection $[24,43,45]$. (Strictly speaking, the boundary $\partial D_{2}$ does not belong to the state space $D_{2}$ for fermions. But since the propagator is zero on this boundary, there is no point in making the distinction between the state space of fermions and bosons as long as the conditions are properly taken into account.)

\section{B. The fermion diffusion process}

In the preceding subsection it was found that the state space $D_{2}$ for two indistinguishable particles is found by imposing an ordering on the configuration space, which introduces an additional boundary. The boundary condition determines the boson or fermion character of the particles because reflection at the boundary leads to a symmetric propagator in configuration space, whereas absorption implies an antisymmetric propagator in configuration space. If one considers $n$ particles, moving freely on a line, one obtains $(n-1)$ extra boundary conditions according to the rule

$$
x_{1} \geqslant x_{2} \geqslant \ldots \geqslant x_{n} \Leftrightarrow\left(x_{1}, x_{2}, \ldots, x_{n}\right) \in D_{n} .
$$

Defining a diffusion process on $D_{n}$ with the appropriate boundary condition, we ensure that the Feynman-Kac functional can be used to incorporate interactions between the particles [4]. Once this is realized, all attention can be given to the process. This means that one has to define the transition probability density to go from an element of $D_{n}$ to another element of $D_{n}$ in one time lapse. This transition probability density of course has to satisfy the conditions for a diffusion process in order to take advantage of the theory developed for multidimensional diffusion on a domain [45]. Knowing the transition probability density, one can construct the sample paths using stationary and independent increments.

For the fermion diffusion process, this transition probability density is a Slater determinant, the elements of which are the single-particle propagators [36]. The transition probability density to go from $\vec{x} \in D_{n}$ to $\bar{x} \in D_{n}$ in a time interval $t$ is

$$
\rho_{F}(\bar{x}, t ; \vec{x}, 0)=\operatorname{det}\left|\rho\left(x_{i}, t ; x_{j}^{\prime}, 0\right)\right|,
$$

where $\rho\left(x_{i}, t ; x_{j}^{\prime}, 0\right)$ is the one-dimensional version of the three-dimensional propagator (2.1). In [26] it was shown that $\rho_{F}$ is a transition probability density for a Markov process on $D_{n}$. This process $\{\widetilde{X}(t) ; t \geqslant 0\}$ can be obtained from the $n$-dimensional process $\{\bar{X}(t) ; t \geqslant 0\}$ given by (2.11) as follows:

$$
X \sim(t)=\left\{\begin{array}{cc}
\bar{X}(t) & \text { for } t \leqslant \tau_{\partial D_{n}} \\
\bar{X}\left(\tau_{\partial D_{n}}\right) & \text { for } t>\tau_{\partial D_{n}}
\end{array}\right.
$$

The Markov time $\tau_{\partial D_{n}}$ is the first exit time of the domain $D_{n}$. It should be emphasized that the algorithm described in [26] to realize the sample paths is based on the present formulation of the process. Another realization of this process can be obtained with the rejection technique [27].

The semigroup property of the transition probability density $\rho_{F}$ follows by using the resolution of the unity operator $1=\int_{D_{n}}|\vec{x}\rangle\langle\bar{x}| d \bar{x}$, whereas the initial condition

$$
\lim _{t \downarrow 0} \rho_{F}(\bar{x}, t ; \vec{x}, 0)=\delta(\bar{x}-\vec{x})
$$

follows from the corresponding property for the onedimensional propagators.

\section{The boson diffusion process}

Like in the case of fermions on a line, the indistinguishability of bosons leads to a state space $D_{n}$ defined in (3.5). This means that $(n-1)$ boundary conditions have to be given to find a process for bosons analogous to fermions. The idea that permutation symmetry can imply an ordering for bosons identical to that for fermions was recently put forward [46] as a consequence of the arbitrariness of the connection between the statistics of the particles and the algebraic properties of the second-quantization operators. Accepting the state space $D_{n}$, the analysis of $D_{2}$ for bosons suggests that reflection at the boundary $\partial D_{n}$ of $D_{n}$ leads to a diffusion process that reflects the Bose-Einstein statistics.

Let $\bar{x}$ and $\bar{y}$ be two elements of $D_{n}$, and construct the following permanent:

$$
\rho_{B}(\bar{x}, t ; \bar{y}, 0)=\operatorname{perm}\left|\rho\left(x_{i}, t ; y_{j}, 0\right)\right| .
$$

It is clear that $\rho_{B}$ is positive for all $(\bar{x}, \bar{y})$ pairs, and that it also satisfies the required initial condition

$$
\lim _{t \downarrow 0} \rho_{B}(\bar{x}, t ; \bar{y}, 0)=\delta(\bar{x}-\bar{y}) .
$$

Furthermore, in order that $\rho_{B}$ can be used as a transition probability density it has to satisfy the conservation of probability and the semigroup property

$$
\int_{D_{n}} \rho_{B}(\bar{x}, t ; \bar{y}, 0) d \bar{y}=1
$$




$$
\int_{D_{n}} \rho_{B}(\bar{x}, t ; \bar{y}, 0) \rho_{B}(\bar{y}, s ; \bar{z}, 0) d \bar{y}=\rho_{B}(\bar{x}, t+s ; \bar{z}, 0) .
$$

The conservation of probability can be derived using the property that a permanent is invariant under an interchange of two rows or columns. Hence

$$
\begin{aligned}
\int_{D_{n}} \rho_{B}(\bar{x}, t ; \bar{y}, 0) d \bar{y} & =\int_{D_{n}} \frac{1}{n !} \sum_{p} \rho_{B}\left(\bar{x}, t ; \overline{y_{p}}, 0\right) d \bar{y} \\
& =\frac{1}{n !} \int_{R_{n}} \operatorname{perm}\left|\rho\left(x_{i}, t ; y_{j}, 0\right)\right| d \bar{y}=1,
\end{aligned}
$$

where use has been made of the fact that $\rho\left(x_{i}, t ; y_{j}, 0\right)$ conserves probability.

The semigroup property follows with an analogous procedure by extending the integration domain $D_{n}$ to $R^{n}$ using the permutation symmetry and subsequently using the semigroup property of the single-particle propagators

$$
\begin{aligned}
& \int_{D_{n}} \rho_{B}(\bar{x}, t ; \bar{y}, s) \rho_{B}(\bar{y}, s ; \bar{z}, 0) d \bar{y} \\
& \quad=\frac{1}{n !} \int_{D_{n}} \sum_{p} \rho_{B}\left(\bar{x}, t ; \overline{y_{p}}, s\right) \rho_{B}\left(\overline{y_{p}}, s ; \bar{z}, 0\right) d \bar{y} \\
& \quad=\frac{1}{n !} \int_{R^{n}} \operatorname{perm}\left|\rho_{B}\left(x_{i}, t ; y_{j}, s\right)\right| \times \operatorname{perm}\left|\rho_{B}\left(y_{j}, s ; z_{k}, 0\right)\right| d \bar{y} \\
& \quad=\operatorname{perm}\left|\rho_{B}\left(x_{i}, t ; z_{k}, 0\right)\right| .
\end{aligned}
$$

In the last step, the semigroup property of the one-particle propagators gives rise to $n$ ! identical contributions.

In order to see how the integration over two permanents leads again to a permanent, the following argument might be useful. Denote by $|\vec{y}\rangle$ a fully symmetrized solution of the Schrödinger equation for free bosons, properly normalized [3]. The resolution of unity is then given by

$$
1=\frac{1}{n !} \int_{R_{n}}|\bar{y}\rangle\langle\bar{y}| d \bar{y}
$$

Denoting by $H_{i}^{0}$ the Hamiltonian for the $i$ th free particle, and by $H^{0}=\sum_{i=1}^{n} H_{i}^{0}$ the Hamiltonian for $n$ free noninteracting bosons, a diffusion from $\bar{z} \in D_{n}$ to $\bar{x} \in D_{n}$ is given by

$$
\begin{aligned}
\rho_{B}(\bar{x}, t ; \bar{z}, 0) & =\left\langle\bar{x}\left|e^{-H^{0} t / \hbar}\right| \vec{z}\right\rangle \\
& =\frac{1}{n !} \int_{R_{n}}\left\langle\bar{x}\left|e^{-H^{0}(t-s) / \hbar}\right| \vec{y}\right\rangle\left\langle\bar{y}\left|e^{-H^{0} s / \hbar}\right| \vec{z}\right\rangle d \bar{y} .
\end{aligned}
$$

Reduction of all identical contributions to the preceding integral by permutation symmetry then leads to

$$
\begin{aligned}
\rho_{B}(\bar{x}, t+s ; \bar{z}, 0) & =\int_{D_{n}}\left\langle\bar{x}\left|e^{-H^{0} t / \hbar}\right| \bar{y}\right\rangle\left\langle\bar{y}\left|e^{-H^{0} s / \hbar}\right| \bar{z}\right\rangle d \bar{y} \\
& =\int_{D_{n}} \rho_{B}(\bar{x}, t ; \bar{y}, 0) \rho_{B}(\bar{y}, s ; \bar{z}, 0) d \bar{y} .
\end{aligned}
$$

Therefore $\rho_{B}(\bar{x}, t ; \bar{y}, 0)$ is a transition probability density to go from $\bar{y}$ to $\bar{x}$ in a time lapse $t$ for a system of noninteracting identical particles with Bose-Einstein statistics. The boundary conditions for this process are determined by the behavior of $\rho_{B}(\bar{x}, t ; \bar{y}, 0)$ at the boundary $\partial D_{n}$. Because $\bar{\nabla} \rho_{B}(\bar{x}, t ; \bar{y}, 0)$ is zero for $\bar{x} \in \partial D_{n}, \rho_{B}$ satisfies Neumann boundary conditions, leading to reflection for the process at the boundary $[4,24,25,42-44]$.

In the mathematical literature the relation between the boson diffusion process and Brownian motion is referred to as the Skohorod equations [24,25]. An account on the relation between Brownian motion and diffusion on a domain with reflecting boundary conditions can be found in [24].

\section{MANY BODY DIFFUSION: A PROCESS FOR IDENTICAL PARTICLES MOVING IN 3D}

In this section we will use the known free-particle density matrix of $n$ fermions and $n$ bosons moving in a $3 n$-dimensional configuration space to analyze its reduction to propagators on a state space in such a way that these propagators are transition probability densities for the processes discussed in the preceding section. The starting point of our analysis is the projection of the density matrix of distinguishable particles on a density matrix which has the correct symmetry properties under permutation of the particle positions:

$$
\rho_{I}(\bar{x}, t ; \vec{x}, 0)=\frac{1}{n !} \sum_{p} \xi^{p} \rho\left(\overline{x_{p}}, t ; \overrightarrow{x^{\prime}}, 0\right) .
$$

The projection operator is a weighted average over all elements $p$ of the permutation group. The weight is the character $\xi^{p}$ of the representation; i.e., $\xi^{p}=1$ for bosons, whereas for fermions $\xi^{p_{+}}=1$ for even permutations $p_{+}$and $\xi^{p_{-}}=-1$ for odd permutations $p_{-}$.

The free-particle density matrix is not a transition probability density: it does not meet the criterion that the process is in a single state in the limit $t \downarrow 0$, i.e., $\lim _{t \downarrow 0} \rho_{I}(\bar{x}, t ; \vec{x}, 0)$ does not satisfy the required initial condition $\delta(\bar{x}-\vec{x})$. For fermions there is the additional complication of the sign problem because $\rho_{I}(\bar{x}, t ; \vec{x}, 0)$ is negative in certain regions of the configuration space. These objections against the interpretation of $\rho_{I}(\bar{x}, t ; \vec{x}, 0)$ as a transition probability density in the configuration space also apply for motions in one dimension. We removed them in the preceding section by restricting the motion to the state space $D_{n}$ with the appropriate boundary conditions.

The questions we have to answer for 3D motion are then what is the appropriate state space and which boundary conditions have to be applied? These questions require a further analysis of $\rho_{I}(\bar{x}, t ; \vec{x}, 0)$. 


\section{A. The permutation symmetry}

Consider a vector $\bar{x}$ in the configuration space $R^{3 n}$, represented as in (2.10) with the $x, y$, and $z$ component of the $j$ th particle as the $(3 j)$ th, $(3 j+1)$ th, and $(3 j+2)$ th component of $\bar{x}$. Its permutations $\bar{x}_{p}$ can be represented as

$$
x \bar{p}^{-}=[p] \bar{x},
$$

where $[p]$ is a $3 n \times 3 n$ dimensional matrix with one $3 \times 3$ identity matrix on each block row and block column, corresponding to each particle. For instance, for 2 particles $[p]$ can take one of both forms $\left[\begin{array}{ll}I & O \\ O & I\end{array}\right]$ or $\left[\begin{array}{ll}O & I \\ I & \bigcirc\end{array}\right]$ with

$$
I=\left[\begin{array}{lll}
1 & 0 & 0 \\
0 & 1 & 0 \\
0 & 0 & 1
\end{array}\right] \text { and } O=\left[\begin{array}{ccc}
0 & 0 & 0 \\
0 & 0 & 0 \\
0 & 0 & 0
\end{array}\right]
$$

The density matrix for the noninteracting identical particles takes the form

$$
\begin{aligned}
\rho_{I}(\bar{x}, t ; \vec{x}, 0)= & \left(\frac{m}{2 \pi \hbar t}\right)^{3 n / 2} \frac{1}{n !} \sum_{p} \xi^{p} \exp \left(-\frac{m}{2 \hbar t}[[p] \bar{x}\right. \\
& \left.-\vec{x}]^{T}[[p] \bar{x}-\vec{x}]\right)
\end{aligned}
$$

which can readily be rewritten as

$$
\begin{aligned}
\rho_{I}(\bar{x}, t ; \vec{x}, 0)= & \left(\frac{m}{2 \pi \hbar t}\right)^{3 n / 2} \exp \left(-\frac{m}{2 \hbar t}(\bar{x} \cdot \bar{x}+\vec{x} \cdot \vec{x})\right) \\
& \times\left(\frac{1}{n !} \sum_{p} \xi^{p} \exp \left(\frac{m}{\hbar t}[p] \bar{x} \cdot \vec{x}\right)\right)
\end{aligned}
$$

\section{B. Projection on even permutations}

We now separate the even permutations $\left\{p_{+}\right\}$from the odd permutations $\left\{p_{-}\right\}$, which can be written as $\left\{p_{-}\right\}=\left\{r p_{+}\right\}$, where $r$ is an element of the permutation group which interchanges two particles. Without loss of generality we take the first and the second particle. (The same element $r$ has to be used for all elements $\left.\left\{p_{-}\right\}\right)$. Using the fact that $\xi^{p_{+}}=1$ for both fermions and bosons, one obtains

$$
\begin{aligned}
\sum_{p} \xi^{p} \exp \left(\frac{m}{\hbar \tau}[p] \bar{x} \cdot \vec{x}\right) \\
=\sum_{p_{+}}\left(\begin{array}{c}
\exp \left(\frac{m}{\hbar \tau}\left[p_{+}\right] \bar{x} \cdot \vec{x}\right) \\
+\xi^{r} \exp \left(\frac{m}{\hbar \tau}[r]\left[p_{+}\right] \bar{x} \cdot \vec{x}\right)
\end{array}\right),
\end{aligned}
$$

where $[r]$ is a $3 n \times 3 n$ matrix whose operation is to interchange the coordinates of the first and the second particle. Hence $[r]$ only differs from the identity matrix in the block column and the block row corresponding to these particles:

$$
[r]=\left[\begin{array}{ccccc}
\bigcirc & I & \bigcirc & \cdots & \bigcirc \\
I & \bigcirc & \bigcirc & \cdots & \bigcirc \\
\bigcirc & \bigcirc & I & \cdots & \bigcirc \\
\vdots & \vdots & \vdots & \ddots & \vdots \\
\bigcirc & \bigcirc & \bigcirc & \cdots & I
\end{array}\right] .
$$

Note that $\xi^{r}=-1$ for fermions and $\xi^{r}=1$ for bosons. Elementary algebra then gives

$$
\begin{aligned}
\sum_{p} \xi^{p} \exp \left(\frac{m}{\hbar \tau}[p] \bar{x} \cdot \vec{x}\right) \\
=\sum_{p_{+}} \exp \left(\frac{1}{2} \frac{m}{\hbar \tau}[\widetilde{I}+r]\left[p_{+}\right] \bar{x} \cdot \vec{x}\right) \\
\quad \times\left(\begin{array}{l}
\exp \left(\frac{1}{2} \frac{m}{\hbar \tau}[\tilde{I}-r]\left[p_{+}\right] \bar{x} \cdot \vec{x}\right) \\
+\xi^{r} \exp \left(-\frac{1}{2} \frac{m}{\hbar \tau}[\tilde{I}-r]\left[p_{+}\right] \bar{x} \cdot \vec{x}\right)
\end{array}\right),
\end{aligned}
$$

where $\widetilde{I}$ denotes the $3 n \times 3 n$ identity matrix (not to be confused with the $3 \times 3$ identity matrix $I)$. Since $[\widetilde{I}+r]$ is invariant if any two particles are interchanged, the permutation symmetry properties of the density matrix are determined by those of

$$
\begin{cases}\cosh \frac{1}{2} \frac{m}{\hbar \tau}[\tilde{I}-r]\left[p_{+}\right] \bar{x} \cdot \vec{x} & \text { for bosons } \\ \sinh \frac{1}{2} \frac{m}{\hbar \tau}[\tilde{I}-r]\left[p_{+}\right] \bar{x} \cdot \vec{x} & \text { for fermions. }\end{cases}
$$

Because

$$
[\tilde{I}-r]=\left[\begin{array}{ccccc}
I & -I & \bigcirc & \cdots & \bigcirc \\
I & I & \bigcirc & \cdots & \bigcirc \\
\bigcirc & \bigcirc & \bigcirc & \cdots & \bigcirc \\
\vdots & \vdots & \vdots & \ddots & \vdots \\
\bigcirc & \bigcirc & \bigcirc & \cdots & \bigcirc
\end{array}\right]
$$

one readily obtains

$$
[\widetilde{I}-r]\left[p_{+}\right] \bar{x} \cdot \vec{x}=\left(\vec{\xi}_{1}-\vec{\xi}_{2}\right) \cdot\left(\vec{x}_{1}^{\prime}-\vec{x}_{2}^{\prime}\right)
$$

where $\vec{\xi}_{1}$ and $\vec{\xi}_{2}$ are the coordinates of the first and second particle in $\left[p_{+}\right] \bar{x}$ :

$$
\left[p_{+}\right] \bar{x}=\left(\begin{array}{c}
\vec{\xi}_{1} \\
\vec{\xi}_{2} \\
\vdots
\end{array}\right)=\left(\begin{array}{c}
\vec{x}_{p_{+}, 1} \\
\vec{x}_{p_{+}, 2} \\
\vdots
\end{array}\right)
$$

The vector $\vec{x}_{j}$ indicates the usual three-dimensional position vector, in contrast to $\bar{x}$, which is a vector of dimension $3 n$, as described above. 


\section{The parity of $\rho_{I}\left(\bar{x}, t ; \bar{x}^{\prime}, 0\right)$ and of its components}

The density matrix of $n$ three-dimensional noninteracting identical particles is given by

$$
\begin{aligned}
\rho_{I}(\bar{x}, t ; \vec{x}, 0)= & \left(\frac{m}{2 \pi \hbar t}\right)^{3 n / 2} e^{\left(-m / 2 \hbar t\left(\bar{x} \cdot \vec{x}+\vec{x}^{\prime} \cdot \vec{x}\right)\right)} \frac{1}{n !} \\
& \times \sum_{p_{+}} e^{m / 2 \hbar \tau[\tilde{I}+r]\left[p_{+} \sqrt{x} \vec{x}\right.} \\
& \times\left\{\begin{array}{r}
2 \cosh \left(\frac{1}{2} \frac{m}{\hbar t}\left(\vec{\xi}_{1}-\vec{\xi}_{2}\right) \cdot\left(\vec{x}_{1}^{\prime}-\vec{x}_{2}^{\prime}\right)\right) \\
2 \sinh \left(\frac{1}{2} \frac{m}{\hbar t}\left(\vec{\xi}_{1}-\vec{\xi}_{2}\right) \cdot\left(\vec{x}_{1}^{\prime}-\vec{x}_{2}^{\prime}\right)\right) \\
\text { for fermions },
\end{array}\right.
\end{aligned}
$$

which is the key result of the present analysis, which allows us to answer the questions above on the state space and its boundary conditions. Indeed, the decompositions

$$
\begin{aligned}
\cosh \vec{a} \cdot \vec{b}= & \cosh a_{x} b_{x} \cosh a_{y} b_{y} \cosh a_{z} b_{z} \\
& +\cosh a_{x} b_{x} \sinh a_{y} b_{y} \sinh a_{z} b_{z} \\
& +\sinh a_{x} b_{x} \cosh a_{y} b_{y} \sinh a_{z} b_{z} \\
& +\sinh a_{x} b_{x} \sinh a_{y} b_{y} \cosh a_{z} b_{z}, \\
\sinh \vec{a} \cdot \vec{b}= & \sinh a_{x} b_{x} \sinh a_{y} b_{y} \sinh a_{z} b_{z} \\
& +\sinh a_{x} b_{x} \cosh a_{y} b_{y} \cosh a_{z} b_{z} \\
& +\cosh a_{x} b_{x} \sinh a_{y} b_{y} \cosh a_{z} b_{z} \\
& +\cosh a_{x} b_{x} \cosh a_{y} b_{y} \sinh a_{z} b_{z}
\end{aligned}
$$

allow us to rewrite the density matrix as a sum of four terms $\rho_{I}(\bar{x}, t ; \vec{x}, 0 ; \ell=0 . .3)$,

$$
\rho_{I}(\bar{x}, t ; \vec{x}, 0)=\sum_{l=0}^{3} \rho_{I}(\bar{x}, t ; \vec{x}, 0 ; \ell),
$$

in which by convention we associate the summation indices $\ell$ as follows with the combinations of given parity with respect to the reflection plane orthogonal to the indicated direction:

Parity of $\rho_{I}(\bar{x}, t ; \vec{x}, 0 ; \ell)$ for bosons

\begin{tabular}{lcccc}
\hline $\begin{array}{l}\text { index } \\
\text { reflection plane }\end{array}$ & $\ell=0$ & $\ell=1$ & $\ell=2$ & $\ell=3$ \\
\hline$\perp x$ & even & even & odd & odd \\
$\perp y$ & even & odd & even & odd \\
$\perp z$ & even & odd & odd & even
\end{tabular}

Parity of $\rho_{I}(\bar{x}, t ; \vec{x}, 0 ; \ell)$ for fermions

\begin{tabular}{lcccc}
\hline $\begin{array}{l}\text { index } \\
\text { reflection plane }\end{array}$ & $\ell=0$ & $\ell=1$ & $\ell=2$ & $\ell=3$ \\
\hline$\perp x$ & & & & \\
$\perp y$ & odd & odd & even & even \\
$\perp z$ & odd & even & odd & even \\
& odd & even & even & odd
\end{tabular}

\section{The orthogonality relations}

The difference in symmetry of these contributions $\rho_{I}(\bar{x}, t ; \vec{x}, 0 ; \ell)$ has important consequences. Consider a function $f\left(\vec{x}^{\prime}\right)$ which is invariant under the permutation of the positions of the particles. It then immediately follows that

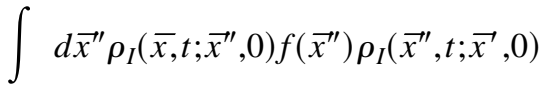

$$
\begin{aligned}
& =\sum_{\ell, \ell^{\prime}=0}^{3} \int d \vec{x}^{\prime \prime} \rho_{I}\left(\bar{x}, t ; \vec{x}^{\prime \prime}, 0 ; \ell\right) f\left(\vec{x}^{\prime \prime}\right) \rho_{I}\left(\vec{x}^{\prime \prime}, t ; \vec{x}, 0 ; \ell^{\prime}\right) \\
& =\sum_{\ell=0}^{3} \int d \vec{x}^{\prime \prime} \rho_{I}\left(\bar{x}, t ; \vec{x}^{\prime \prime}, 0 ; \ell\right) f\left(\vec{x}^{\prime \prime}\right) \rho_{I}\left(\vec{x}^{\prime \prime}, t ; \vec{x}, 0 ; \ell\right),
\end{aligned}
$$

where use has been made of the different parity of the components of $\rho_{I}(\bar{x}, t ; \vec{x}, 0)$ to reduce the double sum into a single summation.

The important consequence is that it is sufficient to analyze each component $\rho_{I}(\bar{x}, t ; \vec{x}, 0 ; \ell)$ with a given parity individually with respect to the interchange of the particles. It is this property which allows us to find a diffusion process for each $\rho_{I}(\bar{x}, t ; \vec{x}, 0 ; \ell)$ separately, as discussed in the next section.

\section{E. The state space for many-body diffusion}

We now analyze each component $\rho_{I}(\bar{x}, t ; \vec{x}, 0 ; \ell)$ of the density matrix separately. For a given value of $\ell$ this function, defined on the configuration space, can be obtained from a transition probability density defined on the state space $D_{n}^{3} \equiv D_{n} \otimes D_{n} \otimes D_{n}$ because it is a product of the transition probabilities of three independent processes, each defined on a $D_{n}$. In other words, the property (4.16) for the density matrix of free identical particles allows us to reduce the configuration space to a much smaller state space with independent fermion or boson processes in each direction.

Let $\left\{\widetilde{X}_{F /}(t) ; t \geqslant 0\right\}$ be the process that generates the sample paths for fermions moving in $3 \mathrm{D}$. Then this process is given according to the following rule:

$$
\tilde{X}_{F}(t)=\left\{\begin{array} { c } 
{ \tilde { X } _ { F } ( t ) } \\
{ \widetilde { Y } _ { F } ( t ) } \\
{ \widetilde { Z } _ { F } ( t ) }
\end{array} \quad \left\{\begin{array} { l } 
{ \widetilde { X } _ { F } ( t ) } \\
{ \widetilde { Y } _ { B } ( t ) } \\
{ \widetilde { Z } _ { B } ( t ) }
\end{array} \quad \left\{\begin{array} { l } 
{ \widetilde { X } _ { B } ( t ) } \\
{ \widetilde { Y } _ { F } ( t ) } \\
{ \widetilde { Z } _ { B } ( t ) }
\end{array} \quad \left\{\begin{array}{c}
\widetilde{X}_{B}(t) \\
\widetilde{Y}_{B}(t) \\
\widetilde{Z}_{F}(t),
\end{array}\right.\right.\right.\right.
$$


where $\widetilde{X}_{F}(t), \widetilde{Y}_{F}(t)$, and $\widetilde{Z}_{F}(t)$ denote fermion diffusion processes in the $x, y$, and $z$ direction. Similarly $\widetilde{X}_{B}(t)$, $Y_{B}^{\sim}(t)$, and $\widetilde{Z}_{B}(t)$ are boson diffusion processes in the $x$, $y$, and $z$ direction. For bosons the decomposition is as follows:

$$
\begin{gathered}
\ell=0 \\
\widetilde{X}_{B}(t)=\left\{\begin{array} { c } 
{ \widetilde { X } _ { B } ( t ) } \\
{ \widetilde { Y } _ { B } ( t ) } \\
{ \widetilde { Z } _ { B } ( t ) }
\end{array} \quad \left\{\begin{array} { c } 
{ \widetilde { X } _ { B } ( t ) } \\
{ \widetilde { Y } _ { F } ( t ) } \\
{ \widetilde { Z } _ { F } ( t ) }
\end{array} \quad \left\{\begin{array} { c } 
{ \widetilde { X } _ { F } ( t ) } \\
{ \widetilde { Y } _ { B } ( t ) } \\
{ \widetilde { Z } _ { F } ( t ) }
\end{array} \quad \left\{\begin{array}{c}
\widetilde{X}_{F}(t) \\
\widetilde{Y}_{F}(t) \\
\widetilde{Z}_{B}(t) .
\end{array}\right.\right.\right.\right.
\end{gathered}
$$

For example, the fermion case with $\ell=1$ is invariant under even permutations $\left\{p_{+}\right\}$of the particle coordinates. Furthermore, under $r$ (interchange of two particles) it is antisymmetric in the $x$ direction and symmetric in the $y$ and $z$ direction. These symmetry properties allow us to restrict the transitions $\vec{x} \rightarrow \bar{x}$ to a domain $D_{n}^{3} \equiv D_{n} \otimes D_{n} \otimes D_{n}$ simultaneously satisfying the conditions

$$
x^{-} \in D_{n}^{3} \Leftrightarrow\left\{\begin{array}{l}
x_{1} \geqslant x_{2} \geqslant \ldots \geqslant x_{n} \\
y_{1} \geqslant y_{2} \geqslant \ldots \geqslant y_{n} \\
z_{1} \geqslant z_{2} \geqslant \ldots \geqslant z_{n}
\end{array}\right.
$$

with the boundary condition that $\rho_{I}(\bar{x}, \vec{x} ; \tau ; \ell=1)$ is zero if during the transition process the boundary $\partial D_{n}$ is hit in the $x$ direction, whereas it is symmetric with respect to the boundary $\partial D_{n}$ in the $y$ and the $z$ direction. This means that an $n$-dimensional fermion diffusion process is required in the $x$ direction, and an $n$-dimensional boson diffusion process in both the $y$ and the $z$ direction.

\section{F. The Feynman-Kac functional}

The many-body process $\left\{\widetilde{X}_{F}(t) ; t \geqslant 0\right\}$ for fermions or $\left\{\widetilde{X}_{B} \ell(t) ; t \geqslant 0\right\}$ for bosons, defined on the state space $D_{n}^{3}$, can be used in the same way as $\{\bar{X}(t) ; t \geqslant 0\}$ to take the interaction between the identical particles into account,

$$
\begin{gathered}
K_{(F, B)}(\bar{x}, t \mid \vec{x})=\sum_{\ell=0}^{3} K_{(F, B) \ell}(\bar{x}, t \mid \vec{x}) \\
K_{(F, B) \ell}(\bar{x}, t \mid \vec{x})=E_{\overline{x^{\prime}}}\left\{f\left[\widetilde{X}_{(F, B) \ell}(\tau)\right] \exp \right. \\
\\
\left.\left[-\frac{1}{\hbar} \int_{0}^{t} V\left(\widetilde{X}_{(F, B) \ell}(s)\right) d s\right]\right\} .
\end{gathered}
$$

Once the propagator of the interacting system on $D_{n}^{3}$ is found with this prescription, the propagator on the configuration space can be obtained from the permutations of the particle indices. The function $f\left[\widetilde{X}_{(F, B)}(\tau)\right]$ expresses again the initial condition. By construction the propagators found on $D_{n}^{3}$ have the correct symmetry with respect to interchanging of the particles. From the positivity of the expressions
$\rho_{I}(\bar{x}, t ; \vec{x}, 0 ; \ell)$ on $D_{n}^{3}$ it follows that $K_{(F, B)}(\bar{x}, t \mid \vec{x})$ is also positive on the same domain.

\section{DISCUSSION AND CONCLUSIONS}

The construction of a state space equipped with a diffusion process to provide sample paths for the Feynman-Kac functional of an interacting system of identical particles takes into account their statistics by imposing for each degree of freedom the appropriate boundary conditions. The spin states are left out of the picture by assuming that there are no spin-dependent interactions involved and therefore the spin as an additional degree of freedom has not to be considered explicitly. Of course the spin degrees of freedom are implicitly present because two identical particles are only considered indistinguishable if they are in the same spin state. The combined process for a particle to diffuse in the configuration space and to change its spin state would require a combination of diffusion and a point process [47]. The latter would change the number of indistinguishable particles, and hence the dimension of the state space. We have only considered the case with a fixed number of indistinguishable particles in the many-body problem. Given a fixed number of fermions or bosons that are not allowed to change their spin degrees of freedom, a process is constructed that takes into account their statistics. All configurations which only differ from each other by a permutation of the coordinates of a particle are represented by the same state. For motion in $1 \mathrm{D}$ this leads to a fermion diffusion process $[26,27]$ and a boson diffusion process, on a state space $D_{n}$ with, respectively, absorption or reflection on the boundary $\partial D_{n}$.

The motion in $3 \mathrm{D}$ combined with the permutation symmetry for $n$ identical objects induces reducible representations in the configuration space. Fortunately the quantum behavior of a noninteracting many-body system is known and could be used to decompose the propagator of such a system into independent boson and fermion processes for each orthogonal direction of the motion. This reduction is the main result of our analysis because it allows us to formulate each process in 3D as four combinations of three independent 1D processes with boundary conditions in the appropriate state space. It is the combination of the boundary conditions on $D_{n} \otimes D_{n} \otimes D_{n}$ that determines whether the process in 3D is suited for fermions or bosons. For example, a 3D process for fermions can contain one 1D fermion diffusion process and two 1D boson diffusion processes. If one wants to calculate the ground state energy of interacting fermions, the two 1D boson processes may be replaced by a Brownian motion in $2 \mathrm{D}$ because the ground state energy of distinguishable particles and the boson ground state energy attain the same value in this limit. This observation explains why we found that the process used formerly $[28,26]$ to study ground state properties of interacting fermions leads to the correct ground state energy [27] (except when a special direction is chosen to define the 1D state space for fermion diffusion, which leads to an excited state for symmetry reasons). The part of the 3D process for fermions that embodies the three 1D fermion diffusion processes will decay faster because the odds to be absorbed are higher. Therefore the contribution of this process to ground state properties should be negligible. 
In the case of bosons one may expect for the same reasonabsorption implies a faster decay-that the low temperature properties can be deduced from the $3 \mathrm{D}$ process that reduces to three $1 \mathrm{D}$ boson processes.

The main achievement of the present approach for bosons is that the sample paths over the configuration space taking into account the permutations $[11,8]$ are replaced by sample paths arising from three independent $1 \mathrm{D}$ processes with well defined boundary conditions. For fermions the significance of our approach is that it provides the solution of the socalled sign problem [7]. Indeed the variance due to the fact that the propagators are antisymmetric under interchange of particle coordinates does not arise in our approach. On the level of functional integration we treat bosons and fermions with the same method. This is not the case in formerly proposed approaches based on the commutation rules for bosons and anticommutation rules for fermions [5,49]. Our approach relies fundamentally on the application of group theoretical concepts to processes, thereby extending previously developed methods for distributions [35] and point processes $[41,40]$.

Summarizing we can say that we introduced the state space together with the process that provides sample paths for the Feynman-Kac functional of a many body problem with a fixed number of identical particles in a given spin state. The propagator over the configuration space can be obtained by the application of permutations to a linear combination of such processes. The boson and fermion diffusion processes are fundamental processes in our approach and their relation to the standard Brownian motion in a domain is settled by the boundary conditions: absorption for the fermion diffusion process and reflection for the boson diffusion process.

\section{ACKNOWLEDGMENTS}

The authors thank J.M.J. Van Leeuwen and W. van Saarloos for a discussion and P. Platzman for discussions and correspondence on the fermion diffusion process. Part of this work has been performed in the framework of the NFWOprojects No. 2.0093.91, 2.0110.91 ("ALPHA-project") and G. 0287.95, and in the framework of the European Community Program Human Capital and Mobility through Contract No. CHRX-CT93-0337. One of the authors (F.B.) acknowledges the National Fund for Scientific Research for financial support.
[1] R. P. Feynman, Rev. Mod. Phys. 20, 367 (1948).

[2] R. P. Feynman and A. R. Hibbs, Quantum Mechanics and Path Integrals (McGraw Hill, New York, 1965).

[3] R. P. Feynman, Statistical Mechanics: A Set of Lectures (W. A. Benjamin, Reading, 1972).

[4] R. Durrett, Brownian Motion and Martingales Analysis (Wadsworth Inc., Belmont, California, 1984).

[5] G. Roepstorff, Path Integral Approach to Quantum Mechanics (Springer, Berlin, 1994).

[6] L. S. Schulman, Techniques and Applications of Path Integration (J. Wiley and Sons, New York, 1981).

[7] D. M. Ceperley, J. Stat. Phys. 63, 1237 (1991).

[8] D. M. Ceperley, Rev. Mod. Phys. 67, 279 (1995).

[9] D. J. Klein and H. M. Pickett, J. Chem. Phys. 64, 4811 (1976).

[10] O. J. Reynolds, D. M. Ceperley, B. J. Alder, and W. A. Chester, J. Chem. Phys. 77, 5593 (1982).

[11] R. P. Feynman, Phys. Rev. 91, 1291 (1953).

[12] F. W. Wiegel, Phys. Rev. B 16, 57 (1975).

[13] E. L. Pollock and D. M. Ceperley, Phys. Rev. B 30, 2225 (1984).

[14] D. M. Ceperley and E. L. Pollock, Phys. Rev. Lett. 56, 351 (1984).

[15] A. P. Lyabartsev and P. N. Vorontsov-Velyaminov, Phys. Rev. A 48, 4075 (1993).

[16] H. F. Jordon and L. D. Fosdick, Phys. Rev. 171, 128 (1968).

[17] B. J. Berne and D. Thirumalai, Ann. Rev. Phys. Chem. 37, 401 (1986).

[18] M. Takahashi and M. Imada, J. Phys. Soc. Jpn. 53, 963 (1984).

[19] S. Zhang and M. H. Kalos, Phys. Rev. Lett. 67, 3074 (1991).

[20] W. H. Newman and A. Kuki, J. Chem. Phys. 96, 1409 (1992).

[21] R. W. Hall, J. Chem. Phys. 97, 6481 (1992).

[22] A. Alavi and D. Frenkel, J. Chem. Phys. 97, 9249 (1992).

[23] A. W. Marshall and I. Olkin, Inequalities: Theory of Majorization and Its Applications (Academic Press, New York, 1979), Chap. 18.
[24] J. M. Harrison and M. I. Reiman, Ann. Prob. 9, 302 (1981).

[25] J. M. Harrison, Brownian Motion and Stochastic Flow Systems (J. Wiley and Sons, New York, 1985).

[26] L. F. Lemmens, F. Brosens, and J. T. Devreese, Phys. Lett. A 189, 437 (1994).

[27] F. Brosens, J. T. Devreese, and L. F. Lemmens, Solid State Commun. 96, 137 (1995).

[28] A. Korzeniowski, J. L. Fry, D. E. Orr, and N. G. Fazleev, Phys. Rev. Lett. 69, 893 (1992); 71, 2160 (1993).

[29] W. M. C. Foulkes, Phys. Rev. Lett. 71, 2158 (1993).

[30] M. Caffarel, D. M. Ceperley, and M. H. Kalos, Phys. Rev. Lett. 71, 2159 (1993).

[31] A. Korzeniowski, J. L. Fry, D. E. Orr, and N. G. Fazleev, Phys. Rev. Lett. 71, 2160 (1993).

[32] For mathematical rigor, one should replace $f[\vec{X}(t)]$ by an event of which the expectation is a $\delta$ function.

[33] W. Fisher, H. Leschke, and P. Müller, J. Phys. Math. Gen. 25, 3835 (1992).

[34] M. Caffarel and P. Claverie, J. Chem. Phys. 88, 1088 (1988); 88, 1100 (1988).

[35] S. Karlin and H. M. Taylor, A Second Course in Stochastic Processes (Academic Press, New York, 1981).

[36] S. Karlin, Total Positivity, Vol. I (Stanford University Press, Stanford, 1968).

[37] H. Bethe, Z. Phys. 71, 205 (1931).

[38] C. Bénard and O. Macchi, J. Math. Phys. 14, 155 (1973).

[39] C. Bénard, Phys. Rev. A 2, 2140 (1970).

[40] O. Macchi, Adv. Appl. Prob. 7, 83 (1975).

[41] D. J. Daley and D. Vere-Jones, An Introduction to the Theory of Point Processes (Springer, Berlin, 1987).

[42] N. G. Van Kampen, Stochastic Processes in Physics and Chemistry (North-Holland, Amsterdam, 1981).

[43] I. I. Gihman and A. V. Skohorod, Stochastic Differential Equations (Springer, Berlin, 1972). 
[44] I. I. Gihman and A. V. Skohorod, The Theory of Stochastic Processes II (Springer, Berlin, 1975).

[45] R. N. Bhattacharya and E. C. Waymire, Stochastic Processes with Applications (J. Wiley \& Sons, New York, 1990).

[46] E. Celeghini, M. Rasetti, and G. Vitiello, J. Phys. A. 28, L239 (1995).
[47] G. F. De Angelis and G. Jona-Lasinio, J. Phys. A 15, 2035 (1982).

[48] This implies that the Feynman-Jensen inequality can be used to estimate the partition function for interacting identical particles.

[49] R. Shankar, Rev. Mod. Phys. 66, 129 (1994). 